\title{
ABSOLUTELY CLOSED SPACES
}

\author{
BY \\ CHEN-TUNG LIU( ${ }^{(1)}$
}

Introduction. The class of Hausdorff absolutely closed spaces was first introduced by Alexandroff and Urysohn [2]. A Hausdorff space $X$ is said to be absolutely closed if every homeomorphic image of $X$ which is a subset of a Hausdorff space $K$, is a closed subspace of $K$. Thus absolute closedness is a slight weakening of compactness. We can find this notion and some extensive studies about it in Alexandroff-Hopf [1] and M. H. Stone [14]. Katětov [10] proved that for any Hausdorff space $X$, there exists a Hausdorff absolutely closed space $\kappa X$ in which $X$ is densely imbedded, and which also has the characteristic property: If $f$ is a continuous mapping from $X$ to a Hausdorff space $T$ such that $f(X)$ is dense in $T$, then there exist a subspace $M$ of $\kappa X$ and a continuous mapping $F$ from $M$ onto $T$ such that $X \subset M$ and $F \mid X=f$.

In the present paper, we extend the definition of absolute closedness to nonHausdorff spaces, and study the corresponding properties of them. In §I, we present a different characterization in terms of extension properties of continuous mappings of $X$ in such a way that $\kappa X$ is the largest absolute closure (see Definition 1.7) of $X$. We also characterize $\kappa X$ completely in the later part of $\S$ I. In $\S I$ I, we show that every space can be imbedded as a closed subspace of an absolutely closed space. In $\S \S I I I$ and IV, we discuss the smallest absolute closure (see Definition 3.2) of a Hausdorff space, and the products of $\kappa X$ 's. Finally, in $\S \mathrm{V}$, we characterize the projective and injective spaces in the category of absolutely closed spaces (with continuous maps).

0. Preliminaries. 0.1. Notations and definitions. The notations used here are standard. For example, we denote the closure of a subset $A$ of a topological space $X$ by $\bar{A}$ or by $\mathrm{Cl}_{X} A$, and the set of all neighborhoods of $p \in X$ by $\mathscr{U}(p)$. We use the standard definitions for filter base, filter, and ultrafilter. An open filter base is a filter base consisting exclusively of open sets. An open filter is a nonempty collection of open sets say $\mathscr{U}$ satisfying the following properties:

(a) $\varnothing \notin \mathscr{U}$.

(b) If $U_{1}, U_{2} \in \mathscr{U}$, then $U_{1} \cap U_{2} \in \mathscr{U}$.

(c) If $U \in \mathscr{U}$ and $G$ is open, $G \supset U$, then $G \in \mathscr{U}$.

An open ultrafilter is an open filter which is maximal in the collection of open filters.

Received by the editors August 16, 1966.

( $\left.{ }^{1}\right)$ The results in this paper are contained in the doctoral thesis written under the direction of Professor A. H. Stone. The author wishes to express her gratitude for his help and instruction. 
0.2. LEMMA. If $\mathscr{U}$ is an open ultrafilter on $X$, the following hold:

(a) If $G$ is an open subset of $X$, then $G \cap U \neq \varnothing$ for all $U \in \mathscr{U}$ iff $G \in \mathscr{U}$.

(b) If $G_{1}, G_{2}$ are open subsets of $X$ and $G_{1} \cup G_{2} \in \mathscr{U}$, then $G_{1} \in \mathscr{U}$ or $G_{2} \in \mathscr{U}$.

(c) If $G_{1} \notin \mathscr{U}$, and $G_{1}$ is open, then $G_{2}=\left(X-\bar{G}_{1}\right) \in \mathscr{U}$.

(d) If $p$ is a cluster point of $\mathscr{U}$, then $\mathscr{U} \rightarrow p$, that is, $\mathscr{U}$ converges to $p$.

Proof. (a), (b), (d) are obvious. For (c), see Obreanu [11]. In (d), note that $\mathscr{U}$ may converge to other points too.

0.3. LEMMA. If $\mathscr{G}$ is an open filter on $X$, and $\mathscr{U}$ is an open ultrafilter containing $\mathscr{G}$, the following hold:

(a) If $p$ is a cluster point of $\mathscr{U}$, then $p$ is a cluster point of $\mathscr{G}$.

(b) If $\mathscr{G} \rightarrow p$, then $\mathscr{U} \rightarrow p$.

Proof. Obvious.

0.4. Lemma. Suppose $X$ is an open $\left({ }^{2}\right)$, dense subset of a topological space $Y$, and $\mathscr{U}$ is an open ultrafilter on $X$. Let $\mathscr{U}^{\prime}=\{G: G$ is open in $Y, G \cap X \in \mathscr{U}\}$. Then $\mathscr{U}^{\prime}$ is an open ultrafilter on $Y$. Moreover, $\mathscr{U} \rightarrow p$ iff $\mathscr{U}^{\prime} \rightarrow p$.

Proof. It is easy to see that $\varnothing \notin \mathscr{U}^{\prime}$ and if $G_{1}, G_{2} \in \mathscr{U}^{\prime}$, then $G_{1} \cap G_{2} \in \mathscr{U}^{\prime}$. Also if $U^{\prime} \in \mathscr{U}^{\prime}$ and $A$ is open in $Y, A \supset U^{\prime}$; then $A \cap X \supset U^{\prime} \cap X \in \mathscr{U}$. Therefore $A \cap X \in \mathscr{U}$, so $A \in \mathscr{U}^{\prime}$. We have so far shown that $\mathscr{U}^{\prime}$ is an open filter on $Y$. Let $\mathscr{G}$ be any open filter on $Y$ such that $\mathscr{U}^{\prime} \subset \mathscr{G}$. We wish to show that $\mathscr{U}^{\prime}=\mathscr{G}$. Take and fix $A \in \mathscr{G}$. It suffices to prove that $A \in \mathscr{U}^{\prime}$. Now for all $U \in \mathscr{U},(A \cap X) \cap U$ $=(A \cap U) \cap X \neq \varnothing$ since $U \in \mathscr{U} \subset \mathscr{G}, A \in \mathscr{G}$, and $X$ is dense in $Y$. Since $\mathscr{U}$ is an open ultrafilter on $X$, then $A \cap X \in \mathscr{U}$. It follows that $A \in \mathscr{U}^{\prime}$.

Now suppose $\mathscr{U} \rightarrow p$. Since $\mathscr{U}$ is an open filter on $Y$ and $\mathscr{U} \subset \mathscr{U}^{\prime}$, then from $0.3(\mathrm{~b})$ it follows that $\mathscr{U}^{\prime} \rightarrow p$.

Conversely, $\mathscr{U}^{\prime} \rightarrow p$ easily implies $\mathscr{U} \rightarrow p$.

0.5. Lemma. Let $X$ be an open, dense subspace of a topological space $Y$. If $\mathscr{U}^{\prime}$ is an open ultrafilter on $Y$ and $\mathscr{U}=\mathscr{U}^{\prime} \cap X=\left\{U^{\prime} \cap X: U^{\prime} \in \mathscr{U}\right\}$, then $\mathscr{U}$ is an open ultrafilter on $X$. Moreover $\mathscr{U} \rightarrow p$ iff $\mathscr{U}^{\prime} \rightarrow p$.

Proof. Since $X$ is a dense, open subset of $Y$, by $0.2(c), X \in \mathscr{U}^{\prime}$. Hence $\mathscr{U}=$ $\left\{U \in \mathscr{U}^{\prime}: U \subset X\right\}$. Clearly $\mathscr{U}$ is an open ultrafilter on $X$ and $\mathscr{U}^{\prime}=\{G: G$ open in $Y, G \cap X \in \mathscr{U}\}$. Hence the last part of the assertion follows from 0.4 .

0.6. LEMma [7, p. 5]. Let $X$ be dense in each of the Hausdorff spaces $S$ and $T$. If the identity mapping on $X$ has continuous extensions $\sigma$ from $S$ into $T$, and $\tau$ from $T$ into $S$, then $\sigma$ is a homeomorphism onto, and $\sigma^{-1}=\tau$.

0.7. Definition. Let $X$ be dense in each of the spaces $S$ and $T$. If $f$ is a homeomorphism from $S$ to $T$ such that $f$ is the identity on $X$, we say $S$ is essentially the same as $T$ relative to $X$.

( ${ }^{2}$ Lemmas 0.4 and 0.5 still hold without assuming that $X$ is open in $Y$. 
0.8. Lemma [7, p. 92]. Let $E$ be a Hausdorff space, and suppose $X$ is dense in $E$. Let $\varphi$ be a continuous mapping from a space $E$ into a space $Y$ such that $\varphi \mid X$ is a homeomorphism; then $\varphi$ maps $E-X$ into $Y-\varphi(X)$.

I. The largest absolute closure of a topological space.

1.1. Definition. A Hausdorff space $X$ is called absolutely closed if $X$ is closed in every Hausdorff space in which it is imbedded.

From this definition, a compact Hausdorff space is absolutely closed.

1.2. Lemma. Let $X$ be a Hausdorff space, then the following are equivalent:

(a) $X$ is absolutely closed.

(b) Every open filter base on $X$ has a cluster point.

(c) Every open cover of $X$ has a finite dense subsystem (whose union is dense in $X)$.

(d) Every open ultrafilter on $X$ converges.

Further, even if $X$ is not Hausdorff, properties (b)-(d) remain equivalent.

Proof. The proof is straightforward. See Bourbaki [4, p. 160], or ScarboroughStone [13].

1.3. Example of an absolutely closed noncompact space (due to Urysohn [15]).

Let $N^{*}=N \cup\{\omega\}$ be the 1-point compactification of $N$, where $N$ is the usual space of the positive integer. Let $K=\left(N \times N^{*}\right) \cup\{\alpha\}$, where $\alpha \notin N \times N^{*}$. The topology on $K$ is defined as follows: $N \times N^{*}$ has the product topology. A basic neighborhood of $\alpha$ is of the form $U_{n}(\alpha)=\bigcup_{i \geqq n} \bigcup_{j \in N}\left\{a_{i j}\right\} \cup\{\alpha\}$ for $n=1,2,3, \ldots$, where $a_{i j}$ is the lattice point $(i, j)$. Then $K$ is easily seen to be absolutely closed from Lemma 1.2(c). However $K$ is not compact, because it has $N \times\{\omega\}$ as a closed subset. This example will be very useful later.

1.4. EXAmple. We may ask, why, in Definition 1.1, we restrict our attention to Hausdorff spaces. As we will observe below that for $T_{1}$ space $X$, " $X$ is closed in every $T_{1}$ space in which it is imbedded" is true only when $X$ is finite.

Let $X$ be an infinite $T_{1}$ space; we can find an infinite sequence of distinct points, say $S=\left\{a_{1}, a_{2}, \ldots, a_{n}, \ldots\right\} \subset X$. Let $p \notin X$ and $Y=X \cup\{p\}$, topologized as follows. For $x \in X$, neighborhoods of $x$ are unchanged. We define a typical neighborhood of $p$, say $U(p)$, as follows: $U(p)=\{p\} \cup\left(\bigcup_{n \geqq n_{0}} U_{n}\left(a_{n}\right)\right)$, where $U_{n}\left(a_{n}\right)$ are neighborhoods of $a_{n}$ in $X$. Then $Y$ is a $T_{1}$ topological space, and $X$ is not closed in $Y$ since $p \in \bar{X}$.

1.5. Definition. Let $X$ be a topological space, not necessarily Hausdorff; we say $X$ is generalized absolutely closed if $X$ satisfies property (b) in 1.2, and hence properties (c) and (d) also.

Scarborough-Stone [13] call such spaces $H(\mathrm{i})$ spaces.

1.6. Definition. Let $X, Y$ be Hausdorff spaces (respectively, arbitrary topological spaces) such that

(a) $X$ is dense in $Y$.

(b) $Y$ is absolutely closed (respectively, generalized absolutely closed). 
We call $Y$ an absolute closure (respectively, generalized absolute closure) of $X$.

1.7. Definition. Let $Y$ be an absolute closure (respectively, generalized absolute closure) of $X$. We say $Y$ is a largest absolute closure (respectively, largest generalized absolute closure) of $X$, if for any other absolute closure (respectively, generalized absolute closure), say $T$ of $X$, and if $i: X \rightarrow T$ is the injection, then there exists $\bar{i}: Y \rightarrow T$ (onto) such that $i \mid X=i$.

Such a $Y$ (if one exists) is essentially unique, as will be shown later.

1.8. THEOREM [5]. If $X=\prod_{\alpha} X_{\alpha}$, where no $X_{\alpha}$ is empty, then $X$ is absolutely closed iff each $X_{\alpha}$ is absolutely closed.

1.9. TheOREM [13]. If $X=\prod_{\alpha} X_{\alpha}$, where no $X_{\alpha}$ is empty, then $X$ is generalized absolutely closed iff each $X_{\alpha}$ is generalized absolutely closed.

1.10. REMARK. It is well known and easy to see that the following hold:

(a) Every continuous image of an absolutely closed space is absolutely closed, if it is Hausdorff.

(b) Finite unions of absolutely closed spaces are absolutely closed, if they are Hausdorff spaces.

(c) The intersection of two absolutely closed spaces may not be absolutely closed.

For the same reasons, (a), (b), (c) hold for generalized absolutely closed spaces.

1.11. THEOREM [11]. Let $X$ be a Hausdorff space; there exists at least one absolute closure $\tilde{X}$ of $X$.

We outline the construction of $\tilde{X}$, as it will be referred to later.

Let $X^{\vee}=\{\mathscr{U}: \mathscr{U}$ is an open nonconvergent ultrafilter on $X\}$. Let $\tilde{X}=X \cup X^{\vee}$ be the disjoint union, under the following topology: The basic open sets of $\tilde{X}$ are those of the form $\tilde{G}=G \cup G^{\vee}$ where $G^{\vee}=\left\{\mathscr{U}: \mathscr{U} \in X^{\vee}, G \in \mathscr{U}\right\}$. Then $X$ is a Hausdorff space such that $X$ is dense in $\tilde{X}$. Moreover, $\tilde{X}$ is absolutely closed.

1.12. THEOREM (KATĚTOV [10]). Let $X$ be a Hausdorff space. Then there exists a Hausdorff absolute closure $Y$ of $X$ satisfying the following property: If $f: X \rightarrow T$ is continuous and $T$ is Hausdorff such that $\mathrm{Cl} f(X)=T$, then there exists a subspace $M$ of $Y$ containing $X$ and a continuous mapping $F$ of $M$ onto $T$ such that $F \mid X=f$.

1.13. TheOREM. For any Hausdorff space $X$, there exists a Hausdorff space $\kappa X$ which is a largest absolute closure of $X$. Moreover, $\kappa X$ is essentially unique in the sense that if $T$ is another largest absolute closure of $X$, then $T$ is essentially the same as $\kappa X$ (see Definition 0.7).

REMARK. $\kappa X$ is the same space as Katětov's absolute closure of $X$ in 1.12, though described differently. The following proof is simpler than Katětov's and takes advantage of Obreanu's construction of $\tilde{X}$ in 1.11.

Proof. We define $\kappa X=X \cup X^{\vee}=\tilde{X}$ as a set as in 1.11. We define $\mathscr{B}$, the basis for the topology of $\kappa X$, as follows: $B \in \mathscr{B}$ iff either $B=G \cup\{\mathscr{P}\}$ where $\mathscr{P} \in X^{\vee}$ 
and $G \in \mathscr{P}$, or $B=G$ where $G$ is open in $X$. It is easy to verify that if $B_{1}, B_{2} \in \mathscr{B}$, then $B_{1} \cap B_{2} \in \mathscr{B}$. Clearly $\mathscr{B}$ is a basis on $\kappa X$. It follows that $X$ is open in $\kappa X$.

We will now show that $\kappa X$ is Hausdorff.

Since $X$ is Hausdorff and $X$ is open in $\kappa X$, then any two distinct points in $X$ have disjoint neighborhoods in $\kappa X$.

Let $x \in X$ and $\mathscr{P} \in X^{\vee}$; then there exists an open neighborhood $G$ of $x$ in $X$ such that $G \notin \mathscr{P}$, for otherwise $\mathscr{P}$ would converge to $x$. Therefore there exists $H \in \mathscr{P}$ such that $H \cap G=\varnothing$. We now have $H \cup\{\mathscr{P}\}$ and $G$ as disjoint neighborhoods of $\mathscr{P}$ and $x$ in $\kappa X$.

Let $\mathscr{P}_{1}, \mathscr{P}_{2}$ be distinct points in $X^{\vee}$; there there exist $G_{1} \in \mathscr{P}_{1}$ and $G_{2} \in \mathscr{P}_{2}$ such that $G_{1} \cap G_{2}=\varnothing$. Let $W_{1}=G_{1} \cup\{\mathscr{P}\}_{1}$ and $W_{2}=G_{2} \cup\{\mathscr{P}\}_{2}$, then $W_{1}$ and $W_{2}$ are disjoint neighborhoods of $\mathscr{P}_{1}$ and $\mathscr{P}_{2}$ in $\kappa X$.

Note that the subspace topology of $X$, as subspace of $\kappa X$, is precisely the given topology of $X$.

$\kappa X$ is absolutely closed: Let $\mathscr{U}$ be an open ultrafilter on $\kappa X$, we want to show $\mathscr{U}$ converges. Put $\mathscr{P}=\{U \cap X: U \in \mathscr{U}\}$. Then by $0.5, \mathscr{P}=\{U \in \mathscr{U}: U \subset X\}$, and $\mathscr{P}$ is an open ultrafilter on $X$. If $\mathscr{P}$ converges to a point $x \in X$, then $x$ is a cluster point of $\mathscr{U}$, hence $\mathscr{U} \rightarrow x$. If $\mathscr{P}$ is nonconvergent on $X$, then $\mathscr{P} \in X^{\vee}$. Let $W=G \cup\{\mathscr{P}\}$ be a neighborhood of $\mathscr{P}$ in $\kappa X$; then $G \in \mathscr{P}$. Therefore $G \in \mathscr{U}$. Thus $G$ meets every member of $\mathscr{U}$. All the more, so does $W$. Hence $\mathscr{P}$ is a cluster point of $\mathscr{U}$ in $\kappa X$, thus $\mathscr{U} \rightarrow \mathscr{P}$.

We want to prove that $\kappa X$ is a largest absolute closure of $X$. Let $T$ be an absolute closure of $X$ and $i: X \rightarrow T$ be the inclusion. We want to extend $i$ to $i: \kappa X \rightarrow T$ continuously. It will follow that if such $i$ exists, $i$ will be onto. For by 1.10(a), $i(\kappa X)$ is absolutely closed, so it is closed in $T$, and contains a dense subset $X$ of $T$; thus $i(\kappa X)=T$.

Let $\mathscr{P} \in X^{\vee}=\kappa X-X$. Let $\mathscr{U}=\{U: U$ open in $T$ and $U \cap X \in \mathscr{P}\}$. By $0.4, \mathscr{U}$ is an open ultrafilter on $T$. Since $T$ is absolutely closed, there exists $p \in T$ such that $\mathscr{U} \rightarrow p$. By 0.4 again, we have $\mathscr{P} \rightarrow p$ in $T$. We now define for each $\mathscr{P} \in X^{\curlyvee}$, $i(\mathscr{P})=p$; and for $x \in X, i(x)=x$.

We shall now show that $i$ is continuous.

Case 1. For $x \in X$, let $W$ be an open neighborhood of $x$ in $T$; then

$$
i^{-1}(W) \cap X=i^{-1}(W)=G
$$

is an open neighborhood of $x$ in $X$, hence is open in $\kappa X$, and $i(G) \subset W$.

Case 2. For $\mathscr{P} \in X^{\vee}$, we have $i(\mathscr{P})=p$ where $\mathscr{P} \rightarrow p$ in $T$. Let $W$ be an open neighborhood of $p$ in $T$; there exists $G \in \mathscr{P}$ such that $G \subset W$. Thus $G \cup\{\mathscr{P}\}$ will be an open neighborhood of $\mathscr{P}$ in $\kappa X$ such that

$$
i(G \cup\{\mathscr{P}\})=i(G) \cup i(\mathscr{P})=i(G) \cup\{p\}=G \cup\{p\} \subset W .
$$

$\kappa X$ is essentially unique: For if $Y$ is an absolute closure of $X$ such that $Y$ is a 
largest such in the sense of 1.7, then there exists $i: Y \rightarrow \kappa X$ where $i$ is a continuous extension of the inclusion map $i: X \rightarrow \kappa X$. Also, there exists $j: \kappa X \rightarrow Y$ such that $j$ is also a continuous extension of the inclusion map $i$. Therefore by $0.6, \kappa X$ is essentially the same as $Y$.

1.14. Definition. If $X$ is a subspace of a topological space $Y$, we say $Y$ is Hausdorff except for $X$ if for any pair of distinct points $a, b$ in $Y$, which are not both in $X, a, b$ have disjoint neighborhoods in $Y$.

1.15. THEOREM. For any topological space $X$, there exists $\kappa X$ which is a largest generalized absolute closure of $X$, and which is Hausdorff except for $X$. Moreover, $\kappa X$ is essentially unique.

Proof. We construct $\kappa X$ in exactly the same way as in 1.13. It is absolutely closed by the same proof as above. As was shown in 1.13 , it is clear that $\kappa X$ is Hausdorff except for $X$.

For the second part of the proof, we want to construct $i: \kappa X \rightarrow T$, where $T$ is any other generalized absolute closure of $X$, which extends the inclusion $i: X \rightarrow T$. The same argument shows that every open ultrafilter $\mathscr{P}$ on $X$ converges to some $p$ in $T$. Since now $T$ is not necessarily Hausdorff, $p$ may not be unique. We now define $i$ as follows: For $x \in X$, we define $i(x)=i(x)=x$; for $\mathscr{P} \in X^{\curlyvee}$, choose $p \in T$ such that $\mathscr{P} \rightarrow p$, and define $i(\mathscr{P})=p$. Exactly the same proof shows that $i$ is continuous.

The uniqueness of $\kappa X$ still holds, by the following lemma.

1.16. Lemma. If $X$ is dense in $T$, and $T$ is Hausdorff except for $X$, and if $f: T \rightarrow T$ is continuous and $f \mid X=$ identity on $X$, then $f=$ identity on $T$.

Proof. If not, there exists $y \in T$ such that $f(y)=z$ and $z \neq y$. Thus $y \notin X$. Since $T$ is Hausdorff except for $X$, there exist $U, V$ which are disjoint neighborhoods of $y$ and $z$ in $T$. By continuity of $f$ at $y, y$ has a neighborhood $W$ such that $f(W) \subset V$. Consider the neighborhood $U \cap W$ of $y$. Because $X$ is dense in $T$, there exists $x \in X \cap U \cap W$, and $f(x)=x$. But $f(x) \in V$ since $x \in W$, so $x \in V \cap U$, which is impossible.

1.17. We proved that for a Hausdorff space $X$, if $Y$ is any absolute closure of $X$, there exists $\bar{i}: \kappa X \rightarrow Y$ which extends the inclusion $i: X \rightarrow Y$ continuously. By analogy to the corresponding property of the Stone-Čech compactification, we ask: If $Y$ is any absolutely closed space and $f: X \rightarrow Y$ is continuous, can $f$ always be extended continuously to $\bar{f}: \kappa X \rightarrow Y$ ? The answer is No, from the following example.

Let $X=N$ be the set of positive integers, which is not absolutely closed. Let $Y=N \times N^{*} \cup\{\alpha\}$ as in 1.3. Then $X$ is mapped homeomorphically into $Y$ by $f(x)=(x, \omega)$ for $x \in X$. Suppose there exists $\bar{f}: \kappa X \rightarrow Y$ such that $\bar{f} \mid X=f$. Then by 0.8 , we have $\bar{f}(\kappa X-X) \subset Y-f(X)=Y-(N \times\{\omega\})$. Choose $\mathscr{P} \in \kappa X-X$; then $\bar{f}(\mathscr{P})=p^{\prime} \in Y-N \times\{\omega\}$. Thus either $p^{\prime} \in N \times N$ or $p^{\prime}=\alpha$. In any case, there exists a 
neighborhood $W$ of $p^{\prime}$ which is disjoint from $N \times\{\omega\}$. (In fact, from the topology of $Y$, every basic neighborhood of $p^{\prime}$ is disjoint from $N \times\{\omega\}$.)

Let $G \cup\{\mathscr{P}\}$ be an open neighborhood of $\mathscr{P}$ in $\kappa X$, where $G \in \mathscr{P}$, then

$$
\bar{f}(G \cup\{\mathscr{P}\})=\bar{f}(G) \cup f(\mathscr{P})=f(G) \cup\left\{p^{\prime}\right\}=(G \times\{\omega\}) \cup\left\{p^{\prime}\right\} .
$$

Thus no neighborhood of $\mathscr{P}$ is carried by $\bar{f}$ into $W$. Thus $\bar{f}$ is not continuous and $f$ has no continuous extension to $\kappa X$.

1.18. However, the following result does hold if we restrict attention to open maps.

THEOREM. Let $X, Y$ be topological spaces and suppose $Y$ is generalized absolutely closed. Let $f: X \rightarrow Y$ be a continuous, open map, then there exists $\bar{f}: \kappa X \rightarrow Y$ such that $\bar{f} \mid X=f$.

Proof. Let us define $\bar{f}: \kappa X \rightarrow Y$ as follows: for $x \in X$, we define $\bar{f}(x)=f(x)$; for $\mathscr{P} \in \kappa X-X$, let $f(\mathscr{P})=\{f(G): G \in \mathscr{P}\}$. Then clearly $f(\mathscr{P})$ is an open filter base on $Y$. Let $\mathscr{U}$ be the open filter on $Y$ generated by $f(\mathscr{P})$; we will show that $\mathscr{U}$ is an open ultrafilter on $Y$. For if not, suppose $\mathscr{U} \cup\{A\}$ where $A \notin \mathscr{U}$ and $A$ is open in $Y$, is contained in some open filter; then $f(G) \cap A \neq \varnothing$ for all $G \in \mathscr{P}$. Thus $f^{-1}(A) \cap G \neq \varnothing$ for all $G \in \mathscr{P}$, thus $f^{-1}(A) \in \mathscr{P}$. Therefore $f\left(f^{-1}(A)\right) \in f(\mathscr{P})$, and $A \in \mathscr{U}$, a contradiction. Thus we proved $f(\mathscr{P})$ generates an open ultrafilter $\mathscr{U}$ on $Y$. Therefore there exists $p^{\prime} \in Y$ such that $\mathscr{U} \rightarrow p^{\prime}$. Notice that there may be more than one such $p^{\prime}$; choose one arbitrarily. Let us define $\bar{f}(\mathscr{P})=p^{\prime}$.

We check that $\bar{f}$ is continuous.

If $\mathscr{P} \in \kappa X-X$ and $f(\mathscr{P})=p^{\prime}$ as described above, let $W$ be an open neighborhood of $p^{\prime}$ in $Y$. Then $W \in \mathscr{U}$ because $\mathscr{U} \rightarrow p^{\prime}$. Hence $W \supset f(G)$ for some $G \in \mathscr{P}$. Thus $G \cup\{\mathscr{P}\}$ will be an open neighborhood of $\mathscr{P}$ in $\kappa X$ such that

$$
\bar{f}(G \cup\{\mathscr{P}\})=\bar{f}(G) \cup \bar{f}(\mathscr{P})=f(G) \cup\left\{p^{\prime}\right\} \subset W .
$$

Thus $\bar{f}$ is continuous at $\mathscr{P}$.

If $x \in X$, let $f(x)=p^{\prime}$, and let $W$, an open neighborhood of $p^{\prime}$ in $Y$, be given. Since $\bar{f}(x)=f(x)=p^{\prime}$, then $G=f^{-1}(W)$ will be an open neighborhood of $x$ in $X$, hence an open neighborhood of $x$ in $\kappa X$, such that $\bar{f}(G)=f(G) \subset W$. Thus $\bar{f}$ is continuous at $x \in X$ also.

1.19. Lemma. Let $X$ be a Hausdorff space and let $T$ be an absolute closure of $X$. Then (a) implies (b):

(a) For each $p \in T-X$, and for each open subset $G$ of $X$ such that $p \in \mathrm{Cl}_{T} G$, $G \cup\{p\}$ is open in $T$.

(b) If $G, H$ are open in $X$ and $G \cap H=\varnothing$, then $\mathrm{Cl}_{T} G \cap \mathrm{Cl}_{T} H \subset X$.

Proof. If $p \in(T-X) \cap\left(\mathrm{Cl}_{T} G \cap \mathrm{Cl}_{T} H\right)$ where $G, H$ are open in $X$ and $G \cap H=\varnothing$, then by (a), $G \cup\{p\}$ and $H \cup\{p\}$ are open neighborhoods of $p$ in $T$. 
Hence $(G \cup\{p\}) \cap(H \cup\{p\})=\{p\}$ will be open in $T$, but this is impossible since $X$ is dense in $T$.

1.20. Lemma. Let $X$ be a Hausdorff space, and let $T$ be an absolute closure of $X$. Then the following are equivalent:

(b) If $G, H$ are open in $X$ and $G \cap H=\varnothing$, then $\mathrm{Cl}_{T} G \cap \mathrm{Cl}_{T} H \subset X$.

(b)' Distinct nonconvergent open ultrafilters on $X$ converge to distinct points in $T-X$.

Proof. (b) $\Rightarrow(\text { b) })^{\prime}$ : Let $\mathscr{P}_{1}, \mathscr{P}_{2}$ be two distinct nonconvergent open ultrafilters on $X$. Then there exists $G_{1} \in \mathscr{P}_{1}$ and $G_{2} \in \mathscr{P}_{2}$ such that $G_{1} \cap G_{2}=\varnothing$. As is shown in the proof of 1.13 , there exist $p_{1}, p_{2} \in T-X$ such that $\mathscr{P}_{1} \rightarrow p_{1}$ and $\mathscr{P}_{2} \rightarrow p_{2}$ in $T$. If $p_{1}=p_{2}$, then since $p_{1} \in \mathrm{Cl}_{T} G_{1}$ and $p_{2} \in \mathrm{Cl}_{T} G_{2}$, it would follow that $p_{1}=p_{2}$ $\in\left(\mathrm{Cl}_{T} G_{1} \cap \mathrm{Cl}_{T} G_{2}\right) \cap(T-X)$. This contradicts (b). Thus $p_{1} \neq p_{2}$.

(b) $\Rightarrow$ (b): Let $G, H$ be open sets in $X$ such that $G \cap H=\varnothing$ and suppose $p \in(T-X) \cap\left(\mathrm{Cl}_{T} G \cap \mathrm{Cl}_{T} H\right)$. Let $\mathscr{U}(p)$ be the open filter base of all open neighborhoods of $p$ in $T$. Let $\mathscr{B}$ be the trace of $\mathscr{U}(p)$ on $X$. Then $\mathscr{B} \cup\{G\}$ has the finite intersection property, so it is contained in an open ultrafilter on $X$, say $\mathscr{G}$. We know $\mathscr{G}$ converges in $T$; since $T$ is Hausdorff, we must have $\mathscr{G} \rightarrow p$ in $T$. Similarly $\mathscr{B} \cup\{H\}$ has the finite intersection property, so it is contained in an open ultrafilter on $X$, say $\mathscr{G}^{\prime}$. For the same reason, $\mathscr{G}^{\prime} \rightarrow p$. By $(\mathrm{b})^{\prime}, \mathscr{G}=\mathscr{G}^{\prime}$; but this is impossible, since $G \cap H=\varnothing$ and $G \in \mathscr{G}, H \in \mathscr{G}^{\prime}$.

1.21. THEOREM. If $T$ is an absolute closure of a Hausdorff space $X$ with the following two properties:

(a) All sets of the form $G \cup\{p\}$, where $G$ is open in $X$ and $p \in \mathrm{Cl}_{T} G-X$, are open in $T$.

(b) $X$ is open in $T$.

Then $T$ is essentially the same as $\kappa X$ (see Definition 0.7). Conversely, $\kappa X$ is an absolute closure of $X$ with these properties.

Proof. It is known that $X$ is open in $\kappa X$. Also it is clear that in $\kappa X$, if $\mathscr{P} \in X^{\curlyvee}$, then $\mathscr{P} \in \mathrm{Cl}_{\kappa X} G$ iff $G \in \mathscr{P}$ for $G$ open in $X$. Therefore $\kappa X$ satisfies (a) and (b).

From Theorem 1.13, we know that there exists $i: \kappa X \rightarrow T$ (onto) such that $i \mid X=i$, the inclusion map. We will show that $i$ is one-one and open, then $i$ will be a homeomorphism which is the identity on $X$.

$i$ is one-one: By 0.8 , we have $i(\kappa X-X) \subset T-X$. Thus we only have to show $i$ is one-one on $\kappa X-X$. Recall $i$ is defined by: For $\mathscr{P} \in X^{\vee}=\kappa X-X, i(\mathscr{P})=p$ where $\mathscr{P} \rightarrow p$ in $T$; if $\mathscr{P}_{1}, \mathscr{P}_{2} \in X^{\vee}$ are such that $\mathscr{P}_{1} \neq \mathscr{P}_{2}$, then $\mathscr{P}_{1}$ and $\mathscr{P}_{2}$ are two distinct open nonconvergent ultrafilters on $X$. From 1.20 together with the assumption, we conclude that $\mathscr{P}_{1}$ and $\mathscr{P}_{2}$ converge to distinct points in $T-X$. Thus if $i\left(\mathscr{P}_{1}\right)=p_{1}$ and $i\left(\mathscr{P}_{2}\right)=p_{2}$, we must have $p_{1} \neq p_{2}$. 
$i$ is open:

Case 1. If $x \in X$ and $G$ is an open neighborhood of $x$ in $X$, then $i(G)=i(G)=G$ which is open in $T$.

Case 2. If $\mathscr{P} \in \kappa X-X$, let $G \cup\{\mathscr{P}\}$ be a basic open neighborhood of $\mathscr{P}$ in $\kappa X$; thus $G \in \mathscr{P}$. Now, $i(G \cup\{\mathscr{P}\})=i(G) \cup \bar{i}(\mathscr{P})=i(G) \cup\{p\}=G \cup\{p\}$. Since $\mathscr{P} \rightarrow p$ in $T$, thus $p \in \mathrm{Cl}_{T} G$. Thus $G \cup\{p\}$ is open in $T$ by assumption.

\section{The imbedding of a topological space as a closed subspace of an absolutely} closed space.

2.1. THEOREM. Let $X$ be a Hausdorff space. Then $X$ can be imbedded as a closed subspace of an absolutely closed space $K$.

Proof. Let us define $K$ as follows: $K=\left(X \times N^{*}\right) \cup X^{\vee}$ as a set, where $N^{*}=N \cup\{\omega\}$ is the 1-point compactification of $N$, and $X^{\vee}=\kappa X-X$ is the set of all nonconvergent open ultrafilters on $X$.

We define a topology on $K$ as follows: as usual, $X \times N^{*}$ has the product topology; i.e., for $(x, n) \in X \times N$, a basic neighborhood of it is of the form $G \times\{n\}$ where $G$ is an open neighborhood of $x$ in $X$. And for $(x, \omega) \in X \times\{\omega\}$, a basic neighborhood of it is of the form $G \times[n, \omega]$ where $G$ is an open neighborhood of $x$ in $X$ and $[n, \omega]$ denotes $\{n, n+1, \ldots, \omega\}$.

Let $\mathscr{P} \in X^{\vee} ; \mathscr{P}$ is an open nonconvergent ultrafilter on $X$. We define a basic neighborhood of $\mathscr{P}$ to be of the form $U(\mathscr{P})=(G \times N) \cup\{\mathscr{P}\}$ where $G \in \mathscr{P}$. Notice that $G$ is an open deleted neighborhood of $\mathscr{P}$ in $\kappa X$.

We are going to prove the following:

1. This is a topology on $K$ : We need only check that for $\mathscr{P} \in X^{\vee}, \mathscr{B}(\mathscr{P})=\{U(\mathscr{P})\}$ forms an open neighborhood system at $\mathscr{P}$.

(a) $\mathscr{B}(\mathscr{P}) \neq \varnothing$ since $X \in \mathscr{P}$. Also $\mathscr{P} \in \bigcap \mathscr{B}(\mathscr{P})$.

(b) Given $\left(G_{1} \times N\right) \cup\{\mathscr{P}\}$ and $\left(G_{2} \times N\right) \cup\{\mathscr{P}\}$, two neighborhoods of $\mathscr{P}$, where $G_{1}, G_{2} \in \mathscr{P}$, then $\left(\left(G_{1} \times N\right) \cup\{\mathscr{P}\}\right) \cap\left(\left(G_{2} \times N\right) \cup\{\mathscr{P}\}\right)=\left(\left(G_{1} \cap G_{2}\right) \times N\right) \cup\{\mathscr{P}\}$ is a basic neighborhood of $\mathscr{P}$ because $G_{1} \cap G_{2} \in \mathscr{P}$.

(c) Given $(G \times N) \cup\{\mathscr{P}\} \in \mathscr{B}(\mathscr{P})$, let $y \in(G \times N) \cup\{\mathscr{P}\}$; then either $y=(x, n)$ $\in G \times N$ or $y=\mathscr{P}$. If $y=(x, n)$ then $G \times\{n\}$ will be a basic neighborhood of $y$ contained in the given one. If $y=\mathscr{P}$, then $(G \times N) \cup\{\mathscr{P}\}$ will be a basic neighborhood of $y$ contained in the given one.

2. $X$ can be imbedded as a closed subset of $K$ : This is clear, because we can identify $X$ with $X \times\{\omega\}$ by mapping $f(x)=(x, \omega)$ for each $x \in X$; $f$ is a homeomorphism, and $X \times\{\omega\}$ is closed in $K$.

3. $K$ is Hausdorff: For points in $X \times N^{*}$, it is clearly so, since $X$ and $N^{*}$ are both Hausdorff. Let $(x, n) \in X \times N$ and $\mathscr{P} \in X^{\vee}$; since $\kappa X$ is Hausdorff, there exists an open neighborhood $G_{1}$ of $x$, and a basic open neighborhood of $\mathscr{P}$, say $G_{2} \cup\{\mathscr{P}\}$ where $G_{2} \in \mathscr{P}$, in $\kappa X$, such that $G_{1} \cap\left(G_{2} \cup\{\mathscr{P}\}\right)=\varnothing$. Thus $G_{1} \cap G_{2}=\varnothing$. Now $G_{1} \times\{n\}$ and $\left(G_{2} \times N\right) \cup\{\mathscr{P}\}$ will be two disjoint neighborhoods of $(x, n)$ and $\mathscr{P}$ in $K$ respectively. Let $\mathscr{P}_{1}, \mathscr{P}_{2} \in X^{\vee}$ where $\mathscr{P}_{1} \neq \mathscr{P}_{2}$. Then there exists $G_{1} \in \mathscr{P}_{1}$ and 
$G_{2} \in \mathscr{P}_{2}$ such that $G_{1} \cap G_{2}=\varnothing$. Thus $\left(G_{1} \times N\right) \cup\left\{\mathscr{P}_{1}\right\}$ and $\left(G_{2} \times N\right) \cup\left\{\mathscr{P}_{2}\right\}$ will be two disjoint neighborhoods of $\mathscr{P}_{1}$ and $\mathscr{P}_{2}$ in $K$.

4. $K$ is absolutely closed. Let $\mathscr{E}$ be an open cover of $K$; we want to find a finite dense subsystem of $\mathscr{E}$. It is clear that we may assume $\mathscr{E}$ consists of basic open sets.

For each $\mathscr{P} \in X^{\curlyvee}=\kappa X-X$, pick $W_{p} \in \mathscr{E}$ such that $\mathscr{P} \in W_{p}$. We can write $W_{p}=\left(G_{p} \times N\right) \cup\{\mathscr{P}\}$ where $G_{p} \in \mathscr{P}$.

For each $x \in X$, look at the sequence $\{x\} \times N^{*}$. Pick $W_{0}(x) \in \mathscr{E}$ such that $(x, \omega) \in W_{0}(x)$. We can write $W_{0}(x)=U_{0}(x) \times\left[n_{x}, \omega\right]$ where $U_{0}(x)$ is an open neighborhood of $x$ in $X$ and $n_{x} \in N$. For each $k=1,2, \ldots, n_{x}-1$, there exists $W_{k}(x) \in \mathscr{E}$ such that $(x, k) \in W_{k}(x)$. We can write $W_{k}(x)=U_{k}(x) \times\{k\}$ where $U_{k}(x)$ is an open neighborhood of $x$ in $X$.

Let $U(x)=U_{0}(x) \cap U_{1}(x) \cap \cdots \cap U_{n_{x}-1}(x)$. Then $U(x)$ is open in $X$ for each $x \in X$. Thus $U(x)$ is open in $\kappa X$ for each $x \in X$.

Consider $\mathscr{C}=\{U(x): x \in X\} \cup\left\{G_{p} \cup\{\mathscr{P}\}: \mathscr{P} \in X^{\vee}\right\}$. This is an open cover of $\kappa X$. Since $\kappa X$ is absolutely closed, there exists a finite subsystem, say

$$
\mathscr{G}=\left\{U\left(x_{1}\right), \ldots, U\left(x_{k}\right), G_{p_{1}} \cup\left\{\mathscr{P}_{1}\right\}, \ldots, G_{p_{m}} \cup\left\{\mathscr{P}_{m}\right\}\right\},
$$

satisfying $\mathrm{Cl}(\cup \mathscr{G})=\kappa X$.

Let us define a family $\mathscr{F}$ as follows:

$$
\begin{aligned}
& \left(G_{p_{1}} \times N\right) \cup\left\{\mathscr{P}_{1}\right\} \\
& \left(G_{p_{2}} \times N\right) \cup\left\{\mathscr{P}_{2}\right\} \\
& \quad \vdots \\
& \left(G_{p_{m}} \times N\right) \cup\left\{\mathscr{P}_{m}\right\} \\
& U\left(x_{1}\right) \times\left[n_{x_{1}}, \omega\right], U\left(x_{1}\right) \times\{1\}, \ldots, U\left(x_{1}\right) \times\left\{n_{x_{1}}-1\right\} \\
& U\left(x_{2}\right) \times\left[n_{x_{2}}, \omega\right], U\left(x_{2}\right) \times\{1\}, \ldots, U\left(x_{2}\right) \times\left\{n_{x_{2}}-1\right\} \\
& \quad \vdots \\
& U\left(x_{k}\right) \times\left[n_{x_{k}}, \omega\right], U\left(x_{k}\right) \times\{1\}, \ldots, U\left(x_{k}\right) \times\left\{n_{x_{k}}-1\right\} .
\end{aligned}
$$

We claim that $\mathrm{Cl}(\bigcup \mathscr{F})=K$.

Proof of the claim. First of all, it is clear that $X \times N$ is dense in $K$. Thus it is enough to show that $\mathrm{Cl}(\bigcup \mathscr{F}) \supset X \times N$. For then $\mathrm{Cl}(\bigcup \mathscr{F}) \supset \mathrm{Cl}(X \times N)=K$.

Let $(x, n) \in X \times N$, we want to prove that for each basic neighborhood $W$ of $(x, n), W \cap(\bigcup \mathscr{F}) \neq \varnothing$. Since $W$ is a basic neighborhood of $(x, n)$, we can write $W=G \times\{n\}$ where $G$ is an open neighborhood of $x$ in $X$, hence open in $\kappa X$. Thus $G$ meets some member of $\mathscr{G}$ because $\mathrm{Cl}(\bigcup \mathscr{G})=\kappa X$. Thus either $G \cap U\left(x_{i}\right) \neq \varnothing$ for some $1 \leqq i \leqq k$ or $G \cap G_{p,} \neq \varnothing$ for some $1 \leqq j \leqq m$.

Case 1. $G \cap U\left(x_{i}\right) \neq \varnothing$ where $1 \leqq i \leqq k$.

(a) If $n \geqq n_{x_{i}}$, then $W=G \times\{n\}$ meets $U\left(x_{i}\right) \times\left[n_{x_{i}}, \omega\right]$ which is a member of $\mathscr{F}$. In fact, $(G \times\{n\}) \cap\left(U\left(x_{i}\right) \times\left[n_{x_{i}}, \omega\right]\right)=\left(G \cap U\left(x_{i}\right)\right) \times\{n\} \neq \varnothing$.

(b) If $n<n_{x_{i}}$, then $1 \leqq n \leqq n_{x_{i}}-1$; thus $W=G \times\{n\}$ meets $U\left(x_{i}\right) \times\{n\}$ which is a member of $\mathscr{F}$. 
Case 2. $G \cap G_{p_{j}} \neq \varnothing$ for some $1 \leqq j \leqq m$, then $W=G \times\{n\}$ meets $\left(G_{p_{j}} \times N\right) \cup\left\{\mathscr{P}_{j}\right\}$ which is a member of $\mathscr{F}$.

Thus we have proved that $K$ is absolutely closed.

2.2. TheOREM. Let $X$ be any topological space. Then $X$ can be imbedded as $a$ closed subspace of a generalized absolutely closed space.

Proof. We know, by Theorem 1.15 , that $\kappa X$, which is the largest generalized absolute closure of $X$, still exists and is constructed in the same way. The previous construction of $K$ in 2.1 still applies, and exactly the same proof shows that $K$ is generalized absolutely closed.

2.3. REMARK. Strecker (unpublished) has shown that every Hausdorff space can be imbedded as a subspace of a minimal Hausdorff space. Combining this with 2.1, we have the following stronger result: Every Hausdorff space can be imbedded as a closed subspace of a minimal Hausdorff space.

III. Smallest absolute closure. For locally compact spaces $X$, we can construct the 1-point compactification $X^{*}$ of $X$ by adding one single point to $X$. It has been shown [6] that if $Y$ is a Hausdorff compactification of a noncompact Hausdorff space $X$ which is minimal (in a natural sense), then $Y$ is essentially the same as $X^{*}$, and moreover, $X$ has to be locally compact.

In this section, we will discuss the analogous theorems for locally absolutely closed spaces. We restrict our attention to Hausdorff spaces in this section.

3.1. Definition. A Hausdorff space $X$ is locally absolutely closed if for each point in $X$, there exists a neighborhood of it which is absolutely closed. From this definition, every absolutely closed space is locally absolutely closed.

3.2. Definition. Let $T$ be an absolute closure of $X$; we say $T$ is a smallest absolute closure of $X$, if for any other absolute closure $Y$ of $X$, there exists $i^{\prime}: Y \rightarrow T$ such that $i^{\prime}$ is a continuous extension of the inclusion $i: X \rightarrow T$.

3.3. REMARK. If $X$ has a smallest absolute closure $T$, then $T$ is essentially unique in the sense of 0.7 .

The following two theorems are quoted from Obreanu's paper [12] about locally absolutely closed spaces.

3.4. THEOREM. For every locally absolutely closed space $X$ which is not absolutely closed, we can construct an absolutely closed space $X^{*}$.by adjoining one single point $\omega$ to $X$, in such a way that $X$ is dense in $X^{*}$ and the trace on $X$ of the open filter of open neighborhoods of $\omega$ is the open filter $\mathscr{B}$, where $\mathscr{B}$ is the open filter generated by the family of all complements of absolutely closed subspaces of $X$.

3.5. THEOREM. In order that we can imbed a space $X$ which is not absolutely closed, into an absolutely closed space from which it differs by only 1 point, it is necessary and sufficient that $X$ be locally absolutely closed.

We are now ready to prove the following theorem. 
3.6. TheOREM. (a) If $X$ is locally absolutely closed, but not absolutely closed, then $X^{*}$ constructed above (see 3.4) is the smallest absolute closure of $X$.

(b) If a space $X$ has a smallest absolute closure say $\omega(X)$, then $X$ is locally absolutely closed and $\omega(X)$ is essentially the same as $X^{*}$, or $X$.

Proof. For (a), let $Y$ be any absolute closure of $X$; we want to show that there exists $i^{\prime}: Y \rightarrow X^{*}$ such that $i^{\prime}$ is an extension of the inclusion $i: X \rightarrow X^{*}$.

Let us define $i^{\prime}(Y-X)=\omega$ and $i^{\prime} \mid X=i$; then $i^{\prime}$ is continuous. For let $W$ be an open neighborhood of $\omega$ in $X^{*}$; then $W \supset G \cup\{\omega\}$ where $G=X-V$, where $V$ is absolutely closed in $X$. Thus

$$
i^{\prime-1}(W) \supset i^{\prime-1}(G \cup\{\omega\})=i^{\prime-1}(G) \cup i^{\prime-1}(\omega)=G \cup(Y-X)=Y-V
$$

which is open in $Y$, since $V$ is absolutely closed and therefore closed in $Y$. Thus $i^{\prime}$ is continuous at each point of $Y-X$. Also, since $X$ is locally absolutely closed in $Y, X$ is open in $Y$ [12]. Thus $i^{\prime}$ is continuous at each point of $X$.

For (b), if $\omega(X)$ is the smallest absolute closure of $X$, we want to show that $\omega(X)=X \cup$ at most 1 point $\}$ and that $X$ is locally absolutely closed. Suppose, on the contrary, there exist $a, b \in \omega(X)-X$ such that $a \neq b$. Since $\omega(X)$ is an absolute closure of $X$, there exists $f: \kappa X \rightarrow \omega(X)$ such that $f$ is a continuous extension of the inclusion $i: X \rightarrow \omega(X)$. Now $f$ is onto (because $f(\kappa X)$ is absolutely closed in $\omega(X)$, hence it is closed in $\omega(X)$, and contains the dense subspace $X$ of $\omega(X))$. Thus there exist $p, q \in \kappa X-X$ such that $f(p)=a$ and $f(q)=b$. Of course $p \neq q$. We identify $p, q$ in $\kappa X$. Let $Z$ be the quotient space of $\kappa X$ under this identification. Let $g: \kappa X \rightarrow Z$ be the quotient map. Thus $g \mid X=$ identity on $X$, and $g(p)=g(q)=[p] \in Z$.

We verify that $Z$ is Hausdorff: If $x, y$ are distinct points in $Z$ such that $x, y \neq[p]$, then $x, y \in \kappa X-\{p, q\}$ which is open in $\kappa X$. There exist $G_{1}, G_{2}$ which are disjoint open neighborhoods of $x, y$ in $\kappa X-\{p, q\}$; hence $G_{1}, G_{2}$ are disjoint open neighborhoods of $x, y$ in $\kappa X$. Thus $g\left(G_{1}\right)=G_{1}$ and $g\left(G_{2}\right)=G_{2}$ will be disjoint neighborhoods of $x, y$ in $Z$. If $x \in Z$ is such that $x \neq[p] \in Z$, let $G, H$ be disjoint open sets in $\kappa X$ such that $G$ contains $x, H$ contains $p$ and $q$. Then $g(G)$ and $g(H)$ will be disjoint open sets in $Z$ such that $x \in g(G)$ and $[p] \in g(H)$.

$X$ is a subspace of $Z$ because $G \cap X=g(G) \cap X$ for any open set $G$ in $\kappa X$.

$Z$ is an absolute closure of $X: Z$ is absolutely closed because it is a continuous image of an absolutely closed space $\kappa X$. Moreover, if $G$ is open in $Z$, then $g^{-1}(G)=G \subset_{\kappa} X$ if $[p] \notin G$; and $g^{-1}(G)=G \cup\{p, q\} \subset_{\kappa} X$ if $[p] \in G$. In any case, $G \cap X \neq \varnothing$, since $X$ is dense in $\kappa X$.

By assumption, there exists $h: Z \rightarrow \omega(X)$ such that $h \mid X=i$ where $i$ is the inclusion map from $X$ into $\omega(X)$. Let $f^{\prime}=h \circ g$, where $\kappa X \stackrel{g}{\rightarrow} Z \stackrel{h}{\rightarrow} \omega(X)$. Thus $f^{\prime}: \kappa X \rightarrow \omega(X)$ is continuous and $f^{\prime} \mid X=i$. Since $f$ agrees with $f^{\prime}$ on a dense subset $X$ of $\kappa X$, and both $\kappa X$ and $\omega(X)$ are Hausdorff, $f \equiv f^{\prime}$. But $f(p)=a \neq b=f(q)$ while

$$
f^{\prime}(p)=h \circ g(p)=h \circ g(q)=f^{\prime}(q),
$$


a contradiction. Thus $\omega(X)=X \cup$ at most 1 point $\}$. By Theorem $3.5, X$ is locally absolutely closed. By the essential uniqueness of the smallest absolute closure of $X$, we hence conclude that $\omega(X)$ is essentially the same as $X^{*}$, unless $X$ is itself absolutely closed, in which case obviously $\omega(X)=X$.

IV. Products of $\kappa X$ 's. Glicksberg [9] has proved that for completely regular spaces $X$, supposing the set $\prod_{\alpha \neq \alpha_{0}} X_{\alpha}$ is infinite for every $\alpha_{0}$, a necessary and sufficient condition that $\beta\left(\prod_{\alpha} X_{\alpha}\right)=\prod_{\alpha} \beta X_{\alpha}$ is that $\prod_{\alpha} X_{\alpha}$ be pseudocompact. In this section, we are going to investigate the analogous situation for the product of $\kappa X$ 's. Before dealing with the general case, it is convenient to deal with the case of two factors.

4.1. THEOREM. Let $X, Y$ be nonempty spaces. Then $\kappa(X \times Y)=\kappa X \times \kappa Y$ iff at least one of the following statements is true:

(a) either $X$ or $Y$ is a finite Hausdorff space;

(b) $\kappa X=X$ and $\kappa Y=Y$.

Proof. (If): Suppose (b) is true, then both $X$ and $Y$ are generalized absolutely closed, therefore $X \times Y$ is generalized absolutely closed [13]. Thus

$$
\kappa(X \times Y)=X \times Y=\kappa X \times \kappa Y .
$$

If (a) is true, we may assume $X$ is finite and Hausdorff, therefore absolutely closed, so $X=\kappa X$. We will show $X \times \kappa Y=\kappa(X \times Y)$.

LEMma. If $X=X_{1} \cup X_{2}$, where $X_{1}, X_{2}$ are closed and disjoint subsets of $X$, then $\kappa X \approx \kappa X_{1} \cup \kappa X_{2}$.

Proof of the lemma. We first establish a 1-1 correspondence between $\kappa X$ and $\kappa X_{1} \cup \kappa X_{2}$ which is the identity on $X=X_{1} \cup X_{2}$ by obtaining a 1-1 correspondence between the nonconvergent open ultrafilters on $X$ and those on $X_{i}$, for $i=1,2$.

Let $\mathscr{P}$ be a nonconvergent open ultrafilter on $X$; thus $\mathscr{P} \in \kappa X-X$. For each $G \in \mathscr{P}, G=G_{1} \cup G_{2}$ where $G_{1}=G \cap X_{1}$ is open in $X_{1}$ and $G_{2}=G \cap X_{2}$ is open in $X_{2}$. Since $X=X_{1} \cup X_{2}$ is a discrote union, then $G_{1} \cap G_{2}=\varnothing$, and $G_{1}, G_{2}$ are open in $X$. By 0.2 , we have either $G_{1} \in \mathscr{P}$ or $G_{2} \in \mathscr{P}$, but not both. Suppose $G_{1} \in \mathscr{P}$. We claim that for any $H \in \mathscr{P}$, writing as above $H=H_{1} \cup H_{2}$ where $H_{1}$ is open in $X_{1}$ and $H_{2}$ is open in $X_{2}$, we have $H_{1} \in \mathscr{P}$. For since $H_{2} \cap G_{1}=\varnothing$ and $G_{1} \in \mathscr{P}$, then $H_{2} \notin \mathscr{P}$; hence by $0.2, H_{1} \in \mathscr{P}$. Thus by 0.5 , the trace of $\mathscr{P}$, say $\mathscr{B}$, on $X_{1}$ will be an open nonconvergent ultrafilter on $X_{1}$ and it will generate all of $\mathscr{P}$; and $\mathscr{B} \in \kappa X_{1}-X_{1}$.

Conversely, let $\mathscr{B} \in\left(\kappa X_{1}-X_{1}\right) \cup\left(\kappa X_{2}-X_{2}\right)$; suppose $\mathscr{B} \in \kappa X_{1}-X_{1}$. Thus $\mathscr{B}$ is a nonconvergent open ultrafilter on $X_{1}$ and $\mathscr{B}$ is thus a base of an open filter $\mathscr{P}$ on $X$ since $X_{1}$ is open in $X$. One easily sees that $\mathscr{P}$ must be a nonconvergent open ultrafilter on $X$. Thus $\mathscr{P} \in \kappa X-X$. If the process described in the previous paragraph is applied to $\mathscr{P}$, it clearly yields $\mathscr{B}$ again. One also observes that

$$
\kappa X_{1} \cap \kappa X_{2}=\varnothing \text {. }
$$


Thus we have shown that the following function is a 1-1 correspondence of $\kappa X_{1} \cup \kappa X_{2}$ onto $\kappa X$.

$$
\begin{array}{lll}
f(x)=x & \text { for } x \in X=X_{1} \cup X_{2}, \\
f(\mathscr{B})=\mathscr{P} & \text { for } \mathscr{B} \in\left(\kappa X_{1}-X_{1}\right) \cup\left(\kappa X_{2}-X_{2}\right) \quad \text { and } \quad \mathscr{P} \in \kappa X-X .
\end{array}
$$

We will show $f$ is a homeomorphism.

Take $\mathscr{B} \in\left(\kappa X_{1}-X_{1}\right) \cup\left(\kappa X_{2}-X_{2}\right)$. Suppose $\mathscr{B} \in\left(\kappa X_{1}-X_{1}\right)$. Let $\mathscr{P}=f(\mathscr{B})$ and let $W=G \cup\{\mathscr{P}\}$ be an open neighborhood of $\mathscr{P} \in \kappa X-X$ where $G \in \mathscr{P}$. Let $G=G_{1} \cup G_{2}$ where $G_{1}$ and $G_{2}$ decompose $G$ into disjoint open sets as described above. By 0.2 , suppose $G_{1} \in \mathscr{P}$. Thus $G_{1} \cup\{\mathscr{B}\}$ will be a neighborhood of $\mathscr{B}$ such that $f\left(G_{1} \cup\{\mathscr{B}\}\right) \subset G \cup\{\mathscr{P}\}$. Therefore $f$ is continuous at each point of

$$
\left(\kappa X_{1}-X_{1}\right) \cup\left(\kappa X_{2}-X_{2}\right) \text {. }
$$

Also $f$ is clearly continuous at each point of $X=X_{1} \cup X_{2}$, since $X$ is open in $\kappa X$. We will now show $f$ is also an open map on $\kappa X_{1} \cup \kappa X_{2}$. Since both $\kappa X_{1}$ and $\kappa X_{2}$ are open in $\kappa X_{1} \cup \kappa X_{2}$, it suffices to show $f$ is open in $\kappa X_{1}$ and $\kappa X_{2}$. Suppose $W=G_{1} \cup\{\mathscr{B}\}$ is a basic open set of $\kappa X_{1}$. Then $G_{1} \in \mathscr{B}$. Hence $f(W)=G_{1} \cup\{\mathscr{P}\}$. Since $\mathscr{B} \subset \mathscr{P}$, then $G_{1} \in \mathscr{P}$. Since $G_{1}$ is open in $X, f(W)=G_{1} \cup\{\mathscr{P}\}$ is open in $\kappa X$. Now if $W \subset X_{1}$, then $W$ is open in $X$ since $X_{1}$ is open in $X$. Hence $f(W)=W$ is open in $\kappa X$. Thus $f$ is open on $\kappa X_{1}$. Similarly, $f$ is open on $\kappa X_{2}$.

Thus we have shown that $\kappa X \approx \kappa X_{1} \cup \kappa X_{2}$, proving the lemma. By using finite induction, we can easily show that if $X=X_{1} \cup \ldots \cup X_{n}$, a discrete union, then $\kappa X \approx \kappa X_{1} \cup \cdots \cup \kappa X_{n}$.

Now we return to the proof of the theorem. Since $X$ is finite, let

$$
X=\left\{x_{1}, x_{2}, \ldots, x_{n}\right\} \text {. }
$$

Thus

$$
X \times Y=\left(\left\{x_{1}\right\} \times Y\right) \cup \cdots \cup\left(\left\{x_{n}\right\} \times Y\right) .
$$

Since $X$ is finite Hausdorff, then by the lemma, we have

$$
\begin{aligned}
\kappa(X \times Y) & \approx \kappa Y_{1} \cup \cdots \cup \kappa Y_{n}, \quad \text { a discrete union } \\
& \approx \kappa Y \cup \cdots \cup \kappa Y, \quad n \text { times, } \\
& \approx \kappa X \times \kappa Y .
\end{aligned}
$$

Thus $\kappa X \times \kappa Y=X \times \kappa Y \approx \kappa(X \times Y)$.

(Only if): Assume $\kappa(X \times Y)=\kappa X \times \kappa Y$ and suppose (b) fails; say $\kappa Y \neq Y$. Let $p \in \kappa Y-Y$. Then

$$
\begin{aligned}
\kappa X \times\{p\} \subset \kappa X \times(\kappa Y-Y) & =(\kappa X \times \kappa Y)-(\kappa X \times Y) \subset(\kappa X \times \kappa Y)-(X \times Y) \\
& =\kappa(X \times Y)-X \times Y,
\end{aligned}
$$

which we know is discrete in its relative topology. Hence $\kappa X$, being homeomorphic to $\kappa X \times\{p\}$, is discrete. Being generalized absolutely closed, it must be finite. Hence $X$ is finite Hausdorff. 
4.2. THEOREM. Let $X_{\alpha}$ be nonempty spaces for all $\alpha$. Then $\kappa\left(\prod_{\alpha} X_{\alpha}\right)=\prod_{\alpha} \kappa X_{\alpha}$ iff at least one of the following two conditions is satisfied.

(a) $\kappa X_{\alpha}=X_{\alpha}$ for all $\alpha$.

(b) There exists $X_{\alpha_{0}}$ which is not generalized absolutely closed. $X_{\alpha}$ is finite and Hausdorff for all $\alpha \neq \alpha_{0}$. Moreover, all but finitely many $X_{\alpha}$ 's have only one point.

Proof. (If): Suppose (a) is true; since $X_{\alpha}=\kappa X_{\alpha}$ for all $\alpha$, then $X_{\alpha}$ is generalized absolutely closed for all $\alpha$, hence $\prod_{\alpha} X_{\alpha}$ is generalized absolutely closed (see [13]). Therefore $\kappa\left(\prod_{\alpha} X_{\alpha}\right)=\prod_{\alpha} X_{\alpha}=\prod_{\alpha} \kappa X_{\alpha}$.

Suppose (b) is true: Let $X=\prod_{\alpha \neq \alpha_{0}} X_{\alpha}$. Then $X$ is finite and Hausdorff. Thus $\kappa X=X$. By Theorem 4.1, we have $\kappa\left(X \times X_{\alpha_{0}}\right)=\kappa X \times \kappa X_{\alpha_{0}}=X \times \kappa X_{\alpha_{0}}$. Since each $X_{\alpha}$ is finite, $\kappa X_{\alpha}=X_{\alpha}$ for all $\alpha \neq \alpha_{0}$. Thus

$$
\kappa\left(\prod_{\alpha} X_{\alpha}\right)=\kappa\left(X \times X_{\alpha_{0}}\right)=X \times \kappa X_{\alpha_{0}}=\left(\prod_{\alpha \neq \alpha_{0}} \kappa X_{\alpha}\right) \times \kappa X_{\alpha_{0}}=\prod_{\alpha} \kappa X_{\alpha} .
$$

(Only if): Suppose $\kappa\left(\prod_{\alpha} X_{\alpha}\right)=\prod_{\alpha} \kappa X_{\alpha}$. Suppose now (a) fails for $\alpha_{0}$, i.e., $X_{\alpha_{0}} \neq \kappa X_{\alpha_{0}}$. We want to show (b) holds. Clearly the index-set has more than one member. Let $X=\prod_{\alpha \neq \alpha_{0}} X_{\alpha}$, then $\kappa\left(X \times X_{\alpha_{0}}\right)=\kappa X \times \kappa X_{\alpha_{0}}$. By Theorem 4.1, either $X$ or $X_{\alpha_{0}}$ is finite and Hausdorff. But $X_{\alpha_{0}}$ is not finite, since it is not generalized absolutely closed. Hence $X$ is finite, showing that (b) holds.

V. Projective and injective spaces in the category of absolutely closed spaces. We know that in the category of compact Hausdorff spaces and continuous maps, $X$ is projective (see 5.1) iff $X$ is extremally disconnected [8], and $X$ is injective (see 5.2) iff $X$ is a retract of a Tychnoff cube. In this section, we are going to investigate the projective and injective spaces in the category of absolutely closed spaces (and of generalized absolutely closed spaces) and continuous maps.

5.1. Definition. Let $\mathscr{C}$ be a category of topological spaces and continuous maps; we assume that all continuous maps are admissible; $X \in \mathscr{C}$ is called projective if, whenever $f: Y \rightarrow Z$ and $g: X \rightarrow Z$ are admissible maps where $Y, Z \in \mathscr{C}$, and $f$ is onto, then there exists admissible map $g^{\prime}: X \rightarrow Y$ such that $g=f \circ g^{\prime}$.

5.2. Definition. Let $\mathscr{C}$ be as in 5.1. $X \in \mathscr{C}$ is called injective if, whenever $f: Z \rightarrow Y$ and $g: Z \rightarrow X$ are admissible maps, where $f$ is $1-1$ and $Y, Z \in \mathscr{C}$, then there exists $g^{\prime}: Y \rightarrow X$ such that $g=g^{\prime} \circ f$.

5.3. THEOREM. In the category of Hausdorff absolutely closed spaces and continuous maps, $X$ is projective iff $X$ is finite.

Proof. If $X$ is finite, then clearly it is projective.

Conversely, if $X$ is projective, we will show that $X$ is discrete. Since $X$ is absolutely closed and discrete, $X$ must be finite.

Suppose, on the contrary, that $X$ is not discrete. Then there exists $a \in X$ such that $a$ is not isolated, i.e., $\{a\}$ is not open. Let $Y=X-\{a\}$, and let $\alpha$ be any point not in $Y \times N^{*}$. We define $A=\left(Y \times N^{*}\right) \cup\{\alpha\}$ with the following topology:

$Y \times N^{*}$ has the product topology. 
Basic neighborhoods of $\alpha$ are of the form $(G \times N) \cup\{\alpha\}$ where $G$ is an open deleted neighborhood of $a$ in $X$. (A deleted neighborhood of $a$ has the form $W-\{a\}$, where $W$ is a usual neighborhood of $a$.) It is easily seen that this determines a topology on $A$.

$A$ is Hausdorff. For points in $Y \times N^{*}$, we can always separate them by disjoint neighborhoods, since $Y$ and $N^{*}$ are both Hausdorff.

Let $(y, n) \in Y \times N$. Since $y \neq a$, there exist $G_{1}, G_{2}$ which are disjoint open neighborhoods of $y$ and $a$ in $X$. Then $G_{1} \times\{n\}$ and $\left(G_{2}^{\prime} \times N\right) \cup\{\alpha\}$, where $G_{2}^{\prime}=G_{2}-\{a\}$, are disjoint neighborhoods of $(y, n)$ and $\alpha$ in $A$.

Let $(y, \omega) \in Y \times\{\omega\}$; then again $y \neq a$. There exist $G_{1}, G_{2}$ which are open neighborhoods of $y$ and $a$ in $X$ such that $G_{1} \cap G_{2}=\varnothing$. Then $G_{1} \times[n, \omega]$ and $\left(G_{2}^{\prime} \times N\right) \cup\{\alpha\}$, where $n \in N$ and $G_{2}^{\prime}=G_{2}-\{a\}$, will be disjoint open neighborhoods of $(y, \omega)$ and $\alpha$ in $A$.

$A$ is absolutely closed. The proof is exactly analogous to the one described in Theorem 2.1 that $K$ is absolutely closed.

Let us define $B=\left(Y \times N^{*}\right) \cup\{\alpha\}$ under the following topology: $Y \times N^{*}$ has the product topology. Basic neighborhoods of $\alpha$ are of the form $\left(G \times N^{*}\right) \cup\{\alpha\}$ where $G$ is an open deleted neighborhood of $a$ in $X$. Let $i: A \rightarrow B$ be the identity map. Then $i$ is clearly continuous; thus $B$ is absolutely closed because it is a continuous image of $A$ (see 1.10). Of course $i$ is onto. Let $j: X \rightarrow B$ be defined by $j(a)=\alpha$ and $j(y)=(y, \omega)$ for each $y \in Y$.

$j$ is continuous. Let $(y, \omega) \in Y \times\{\omega\} \subset B$ and let $W$ be a basic open neighborhood of $(y, \omega)$ in $B$. We may write $W=G \times[n, \omega]$ where $G$ is an open neighborhood of $y$ in $Y$ and $n \in N$. Thus $j(G)=G \times\{\omega\} \subset W$. Let $\alpha \in B ; a=j^{-1}(\alpha)$. Take $W$ a basic neighborhood of $\alpha$ in $B$. Then $W=G \times N^{*} \cup\{\alpha\}$ where $G$ is an open deleted neighborhood of $a$ in $X$. Thus $G \cup\{a\}$ will be an open neighborhood of $a$ in $X$ such that $j(G \cup\{a\})=j(G) \cup j(a)=(G \times\{\omega\}) \cup\{\alpha\} \subset\left(G \times N^{*}\right) \cup\{\alpha\}$. Now, consider the diagram:

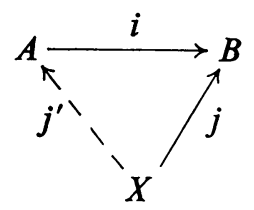

Since $X$ is projective, there exists $j^{\prime}: X \rightarrow A$ such that $j^{\prime}$ is continuous and $i \circ j^{\prime}=j$. Therefore $j^{\prime}(Y)=Y \times\{\omega\}$ and $j^{\prime}(a)=\alpha$. We will show that $j^{\prime}$ is not continuous at $a$ : for if $W$ is any basic open neighborhood of $\alpha$ in $A$, then $W=(G \times N) \cup\{\alpha\}$ for some $G$ open in $Y$. But there exists no open neighborhood $G^{\prime}$ of $a$ in $X$ such that $j^{\prime}\left(G^{\prime}\right) \subset W$, because $j^{\prime}\left(G^{\prime}\right)=G^{\prime} \times\{\omega\} \notin W$. Thus we reach a contradiction. Hence $X$ is discrete and thus finite.

5.4. THEOREM. In the category of generalized absolutely closed spaces and continuous maps, the projective spaces are precisely the finite Hausdorff spaces. 
Proof. If $X$ is projective, generalized absolutely closed, we will show $X$ is discrete. Thus it follows $X$ is finite and Hausdorff. We will sketch the proof and leave the details to the reader.

Let $A \subset X$ be any subset of $X$ and $B=X-A$. We will show $A, B$ are both closed in $X$; it follows then that $X$ is discrete. Let us construct $Y$ as follows: $Y=K_{A} \cup K_{B}$ as a discrete union, where $K_{A}=\left(A \times N^{*}\right) \cup(\kappa A-A)$, disjoint union, under the following topology: $A \times N^{*}$ has the product topology. Let $\mathscr{P} \in \kappa A-A$; a typical neighborhood of $\mathscr{P}$ is of the form $(G \times N) \cup\{\mathscr{P}\}$ where $G$ is open in $A$ and $G \in \mathscr{P}$. Similarly we define $K_{B}$. By Theorem $2.2, K_{A}, K_{B}$ are generalized absolutely closed, and $A \times\{\omega\}$ is a closed subspace of $K_{A}, B \times\{\omega\}$ is a closed subspace of $K_{B}$. Thus $Y$ is generalized absolutely closed by 1.10 .

Let us construct $Z$ as follows: $Z=\left(X \times N^{*}\right) \cup(\kappa A-A) \cup(\kappa B-B)$, where these sets are considered to be disjoint, under the following topology: $X \times N^{*}$ has the product topology. For $\mathscr{P} \in \kappa A-A$, a typical basic neighborhood of $\mathscr{P}$ is of the form $(G \times N) \cup\{\mathscr{P}\}$, where $G$ is open in $X$, and such that $G \cap A=H \in \mathscr{P}$. Similarly for points in $\kappa B-B$. It is easy to see that $Z$ is a topological space. Let $i: Y \rightarrow Z$ be the identity map. $i$ is continuous (the proof is straightforward); therefore $Z$ is generalized absolutely closed. Consider $j: X \rightarrow Z$ defined by $j(x)=(x, \omega)$ for each $x \in X . j$ is clearly continuous. Now look at the following diagram:

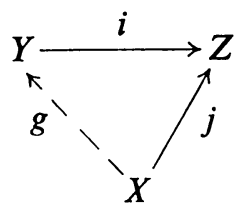

By assumption, $X$ is projective; there exists $g: X \rightarrow Y$, continuous and $i \circ g=j$. Since $i$ is the identity map, and $j(A)=A \times\{\omega\}, j(B)=B \times\{\omega\}$, we conclude that $g(A)=A \times\{\omega\}$ and $g(B)=B \times\{\omega\}$. Therefore $g^{-1}(A \times\{\omega\})=A$ and $g^{-1}(B \times\{\omega\})=B$. We conclude that $A$ and $B$ are closed in $X$ since $A \times\{\omega\}$ and $B \times\{\omega\}$ are closed in $Y$. Thus $X$ is discrete; therefore it is finite and Hausdorff.

5.5. THEOREM. In the category of absolutely closed spaces and continuous maps, the injective spaces are those which consist of only a single point.

Proof. Let $Z=\left\{a_{i j}, b_{i j}, c_{i}, \alpha, \beta: i=1,2, \ldots, j=1,2, \ldots\right\}$ where all these elements are assumed to be distinct.

Define the following neighborhood system on $Z$ : Each $a_{i j}$ is isolated and each $b_{i j}$ is isolated. We define the basic neighborhoods of $c_{i}, \alpha, \beta$ as follows:

$$
\begin{aligned}
& \mathscr{B}\left(c_{i}\right)=\left\{V^{n}\left(c_{i}\right)=\bigcup_{j=n}^{\infty}\left\{a_{i j}, b_{i j}, c_{i}\right\}: n=1,2, \ldots\right\}, \\
& \mathscr{B}(\alpha)=\left\{V^{n}(\alpha)=\bigcup_{j=1}^{\infty} \bigcup_{i=n}^{\infty}\left\{a_{i j}, \alpha\right\}: n=1,2, \ldots\right\}, \\
& \mathscr{B}(\beta)=\left\{V^{n}(\beta)=\bigcup_{j=1}^{\infty} \bigcup_{i=n}^{\infty}\left\{b_{i j}, \beta\right\}: n=1,2, \ldots\right\} .
\end{aligned}
$$


Then $Z$ is minimal Hausdorff, hence absolutely closed (see [3]). (This example is also due to Urysohn [15].)

Let $Y=\left\{a_{i j}, c_{i}, \alpha, \beta: i=1,2, \ldots, j=1,2, \ldots\right\}$, as a subspace of $Z$. Then $Y$ is absolutely closed because $Y=A \cup B$ where $A=\left\{a_{i j}, c_{i}, \alpha: i=1,2, \ldots, j=1,2, \ldots\right\}$ and $B=\{\beta\}$ as subspaces of $Z$ which are both absolutely closed.

Now, if $X$ is absolutely closed and injective, suppose there exists $a, b \in X$ such that $a \neq b$. Since $X$ is Hausdorff, there exist $G_{1}, G_{2}$ such that $G_{1}$ is an open neighborhood of $a$ in $X, G_{2}$ is an open neighborhood of $b$ in $X$, and $G_{1} \cap G_{2} \neq \varnothing$.

Now let us define $f: Y \rightarrow X$ as follows: $f(A)=a$ and $f(\beta)=b$. Then $f$ is continuous, because $A, B$ are open in $Y$. Let $j: Y \rightarrow Z$ be the injection; of course $j$ is one-toone and continuous. Consider the following diagram:

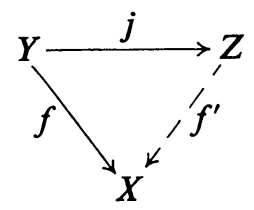

Now, since $X$ is injective, there exists $f^{\prime}: Z \rightarrow X$ such that $f^{\prime}$ is continuous and $f^{\prime} \circ j=f$. Thus $f^{\prime}(A)=a$ and $f^{\prime}(B)=f(\beta)=b$. Hence $f^{\prime-1}\left(G_{1}\right) \supset f^{\prime-1}(a) \supset A$,

$$
f^{\prime-1}\left(G_{2}\right) \supset f^{\prime-1}(b) \supset B \text { and } f^{\prime-1}\left(G_{1}\right) \cap f^{\prime-1}\left(G_{2}\right)=\varnothing .
$$

Thus $f^{\prime-1}\left(G_{1}\right)$ and $f^{\prime-1}\left(G_{2}\right)$ are disjoint open sets containing $A$ and $B$ respectively. But in $Z$, the sets $A, B$ do not have disjoint open neighborhoods. This is a contradiction. Thus $X=\{$ a single point $\}$. Obviously, if $X=\{$ a single point $\}$, then $X$ is injective.

\section{BIBLIOGRAPHY}

1. P. Alexandroff and H. Hopf, Topologie. I, Die Grundlehren der Mathematischen Wissenschaften in Einzeldarstellungen, Bd. 45, Springer, Berlin, 1935.

2. P. Alexandroff and P. Urysohn, Mémoire sur les espaces topologiques compacts, Verh. Nederl. Akad. Wetensch. Afd. Natuurk. Sect. I 14 (1929), 1-96.

3. M. P. Berri, Minimal topological spaces, Trans. Amer. Math. Soc. 108 (1963), 97-105.

4. Bourbaki, Topologie générale, 4th ed., Actualités Sci. Ind., No. 1142, Hermann, Paris, 1965.

5. C. Chevalley and O. Frink, Bicompactness of Cartesian products, Bull. Amer. Math. Soc. 47 (1941), 612-614.

6. A. Dickinson, Compactness conditions and uniform structure, Amer. J. Math. 75 (1953), 224-228.

7. L. Gillman and M. Jerison, Rings of continuous functions, Van Nostrand, Princeton, N. J., 1960.

8. A. M. Gleason, Projective topological spaces, Illinois J. Math. 2 (1958), 482-489.

9. I. Glicksberg, Stone-Čech compactification of products, Trans. Amer. Math. Soc. 90 (1959), 369-382.

10. M. Katětov, Uber H-abgeschlossene und bikompakte Räume, Časopis Pěst. Mat. Fys. 69 (1940), 36-49. 
11. P. Obreanu, Asupra unei probleme a lui Alexandrov si Urysohn, Acad. R. P. Romîne An. Bul. Ști. Ser. Mat. Fiz. Chim. 2 (1950), 101-108.

12. —, Spatii separate minimale, Acad. R. P. Romîne An. Sect. Şti. Mat. Fiz. Chim. Ser. A 3 (1950), 325-349.

13. C. T. Scarborough and A. H. Stone, Products of nearly compact spaces, Trans. Amer. Math. Soc. 124 (1966), 131-147.

14. M. H. Stone, Application of the theory of Boolean rings to general topology, Trans. Amer. Math. Soc. 41 (1937), 375-481.

15. P. Urysohn, Uber die Mächtigkeit der Zusammenhängenden Mengen, Math. Ann. 94 (1925), 266-295.

\author{
UNIVERSITY OF ROCHESTER, \\ ROCHESTER, NeW YORK \\ Vassar College, \\ PoughKeEpsie, New York
}

